\title{
Independent component and pathway-based analysis of miRNA-regulated gene expression in a model of type 1 diabetes
}

Claus H Bang-Berthelsen ${ }^{1,2+}$, Lykke Pedersen ${ }^{3+}$, Tina Fløyel ${ }^{1,2}$, Peter H Hagedorn ${ }^{4,7}$, Titus Gylvin ${ }^{5}$, Flemming Pociot ${ }^{1,2,6^{*}}$

\begin{abstract}
Background: Several approaches have been developed for miRNA target prediction, including methods that incorporate expression profiling. However the methods are still in need of improvements due to a high false discovery rate. So far, none of the methods have used independent component analysis (ICA). Here, we developed a novel target prediction method based on ICA that incorporates both seed matching and expression profiling of miRNA and mRNA expressions. The method was applied on a cellular model of type 1 diabetes.

Results: Microrray profiling identified eight miRNAs (miR-124/128/192/194/204/375/672/708) with differential expression. Applying ICA on the mRNA profiling data revealed five significant independent components (ICS) correlating to the experimental conditions. The five ICs also captured the miRNA expressions by explaining $>97 \%$ of their variance. By using ICA, seven of the eight miRNAs showed significant enrichment of sequence predicted targets, compared to only four miRNAs when using simple negative correlation. The ICs were enriched for miRNA targets that function in diabetes-relevant pathways e.g. type 1 and type 2 diabetes and maturity onset diabetes of the young (MODY).
\end{abstract}

Conclusions: In this study, ICA was applied as an attempt to separate the various factors that influence the mRNA expression in order to identify miRNA targets. The results suggest that ICA is better at identifying miRNA targets than negative correlation. Additionally, combining ICA and pathway analysis constitutes a means for prioritizing between the predicted miRNA targets. Applying the method on a model of type 1 diabetes resulted in identification of eight miRNAs that appear to affect pathways of relevance to disease mechanisms in diabetes.

\section{Background}

microRNAs (miRNAs) are a class of small non-coding RNAs that function as posttranscriptional regulators of gene expression by mediating translational inhibition or mRNA degradation. miRNA bind to "seed" sites, i.e. stretches of 6-8 nucleotides in the 3' untranslated region (UTR) of their target mRNAs. miRNAs regulate various cellular processes and appear to be involved in the development of many diseases.

Most approaches for miRNA target identification rely on either one or a combination of seed matching, site

\footnotetext{
* Correspondence: fpoc0001@glo.regionh.dk

+ Contributed equally

'Glostrup Research Institute, Glostrup University Hospital, DK-2600 Glostrup, Denmark

Full list of author information is available at the end of the article
}

accessibility and phylogenetic conservation [1]. In addition, some have incorporated target site location, multiple target sites and expression profiling [1]. A number of target prediction methods use expression profiling of both miRNA and mRNA expressions [2-5]. Most of the approaches are based on negative correlation, i.e. reciprocal expressions of miRNAs and their degraded target mRNAs.

High-dimensional biological data, such as microarray profiling data, are often interpreted as being composed of sets of transcriptional- or activity programs that explain some, or most, of the complexity in the data [6-8]. Various methods are being applied on profiling data e.g. principal component analysis (PCA) and clustering. In the last couple of years independent component analysis (ICA) has been extensively applied on

\section{(Ciomed Central}


mRNA profiling data and recently, ICA was applied on miRNA profiling data as well [9].

ICA is a computational method for separating mixed independent signals and can be used to decompose the expression matrix into independent components [10]. This decomposition has been shown to be informative in several studies [11-15], and superior to clustering and PCA [16-19]. Apparently, the representation of gene expression as a mix of independent, possibly overlapping, transcriptional programs captures the differential regulation of well-defined biological processes and metabolic pathways $[19,20]$.

Type 1 diabetes (T1D) is an immune-mediated disease characterized by insulin deficiency due to a specific destruction of the pancreatic $\beta$-cells. Proinflammatory cytokines are involved in the destruction through induction of apoptosis [21]. The endocrine cells in the pancreatic islets all arise from the same progenitor stem cell and the maturation of the different cell types is dependent on a number of factors, such as transcription factors and miRNAs, that are activated in a tightly regulated pattern [22,23]. One of the central transcription factors in pancreatic development is Pancreatic and duodenal homeobox $1(\mathrm{Pdx}-1)$ [24-26]. In the mature endocrine cells, Pdx-1 expression is restricted to the $\beta$-cell, where it is important for insulin expression [27].

In the present study, we have used a model of type 1 diabetes based on $\beta$-cell maturation and interleukin$1 \beta$ (IL-1 $1 \beta$ ) sensitivity. In response to $\mathrm{Pdx}-1$ induction the cells progress from an immature to a mature $\beta$-cell phenotype [28]. The $\beta$-cell maturation is accompanied by an increased sensitivity to the toxic effects of IL-1 $\beta$ that is reflected in both transcriptional and protein expression patterns [29-31]. Genes regulated by Pdx1 are therefore believed to be involved in the acquired IL-1 $\beta$ sensitivity, and identification of these genes would provide knowledge about the mechanisms underlying this $\beta$-cell specific trait. Interestingly, a study investigating genomic targets of transcription factors in a $\beta$-cell line suggested that several miRNAs are under $\mathrm{Pdx}-1$ regulation [32]. Furthermore, a number of miRNAs have been implicated in the regulation of pancreatic development and $\beta$-cell differentiation [22,33-36].

Here, we have developed a novel miRNA target prediction method that is based on ICA and combines seed matching and expression profiling. We comprehensively profiled both miRNA and mRNA expressions from the type 1 diabetes model. ICA was applied on the mRNA expression data for identification of miRNA targets. Our method was compared to negative correlation. We validated our observations by use of pathway analysis and human pancreatic islet preparations.

\section{Results}

\section{$\beta$-cell specific gene expression}

To confirm that the INSr $\alpha \beta$ cells progressed from a hybrid $\alpha \beta$-like phenotype to a more mature $\beta$-cell phenotype upon $\mathrm{Pdx}-1$ induction as seen in previous studies $[28,37]$, the expression levels of known insulin and Pdx1 dependent genes were examined using the array data (Additional file 1). The observed expression profiles were in agreement with insulin and $\mathrm{Pdx}-1$ regulations known from other studies.

The dox-induced $\mathrm{Pdx}-1$ expression was examined using $\mathrm{qPCR}$ and western blotting with mouse-specific primers and antibody, respectively. We found that dox treatment for 24 hours resulted in increased expression of both Pdx-1 mRNA (33 fold, $\mathrm{p}<0.05$ ) and protein (6 fold, $\mathrm{p}<0.05)$ (Figure 1).

\section{miRNAs differentially expressed in a model of type 1 diabetes}

miRNA expression profiling resulted in identification of eight miRNAs with differential expression in response to Pdx-1 induction and/or IL-1 $\beta$ exposure. All eight miRNAs (miR-124/128/192/194/204/375/672/708) showed significant $(\mathrm{p}<0.05)$ changes in expression between conditions and/or time points (Figure 2A). The eight miRNA expression profiles capture all three experimental conditions: dox-induced $\mathrm{Pdx}-1$ expression, IL-1 $\beta$ exposure and time. For example, the miR672 expression decreased significantly $(\mathrm{p}<0.05)$ with time independent of Pdx-1 induction and/or IL- $1 \beta$ treatment. The reversed pattern was seen for miR-204, though only for cells with induced Pdx-1 expression.

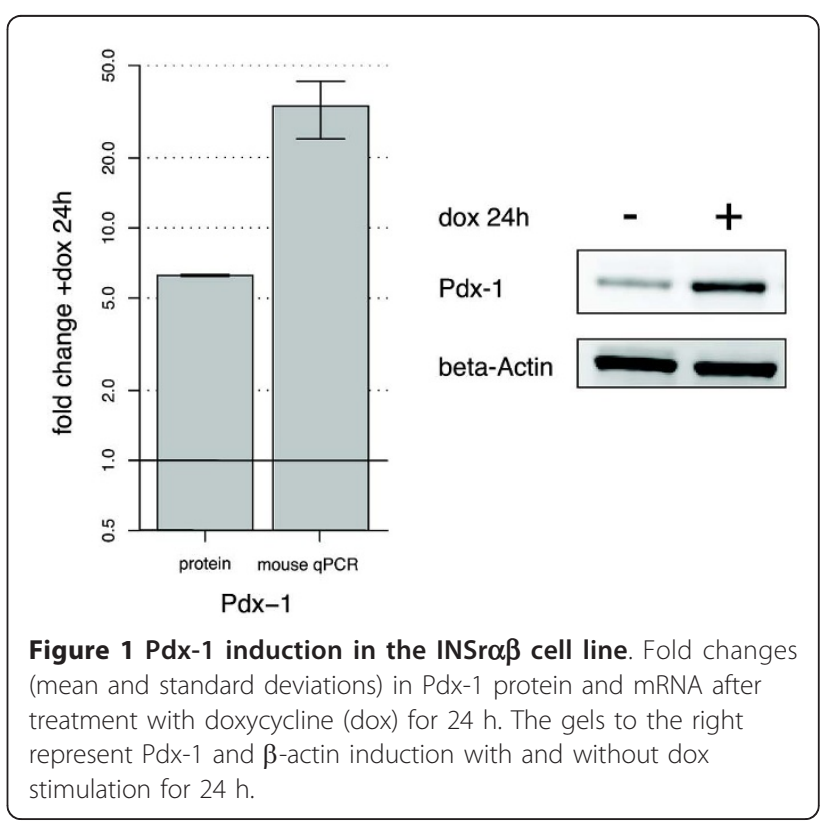



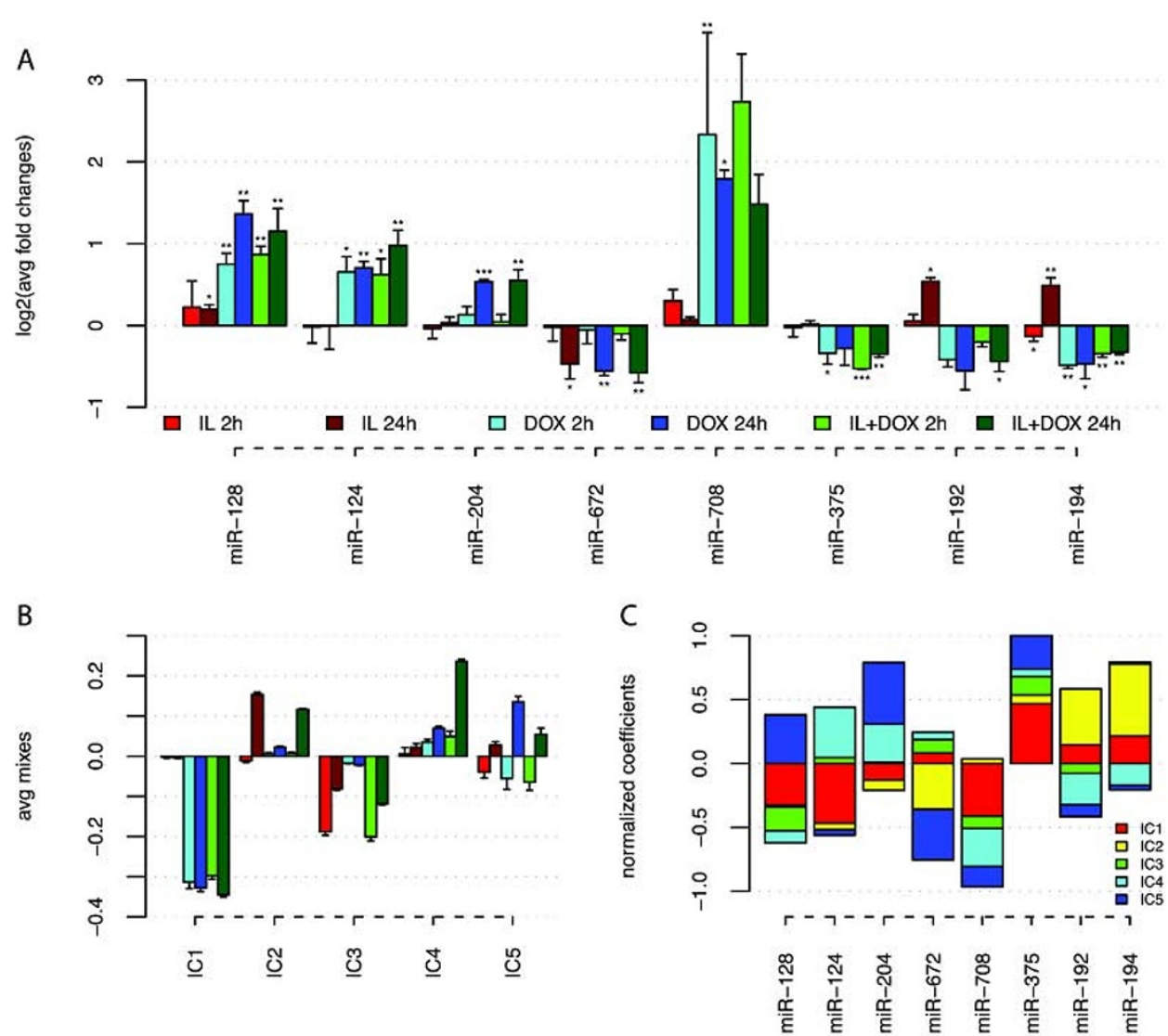

Figure 2 miRNA expressions and mixes of independent components. There are three experimental conditions: Pdx-1 induction (dox treatment), IL-1 $\beta$ treatment and time (samples are taken $2 \mathrm{~h}$ and $24 \mathrm{~h}$ after treatment). (A) Log2-transformed fold changes (mean and standard deviations) between experimental and control (untreated cells) conditions. *: $0.05>q>0.01, * *: 0.01>q>0.001, * * * 0.001>q>0$. (B) For each independent component (IC) the average of mixes are shown for each condition. Bars represent mean and standard deviation. IC 1 is a $\mathrm{Pdx}-1$ component showing mixes correlating to Pdx-1 induction. IC 2 and 3 are cytokine components with mixes correlating to IL-1 $\beta$ treatment. IC 4 shows mixes correlating to induction of Pdx-1 and treatment with IL-1 $\beta$ after $24 \mathrm{~h}$. IC 5 has mixes increasing from $2 \mathrm{~h}$ to $24 \mathrm{~h}$ in all three conditions. (C) The coefficient for the linear combination of the ICs giving the best fit of the miRNA expressions. The coefficients are scaled to have an absolute sum of one.

A third example was the up-regulating effect of IL-1 $\beta$ treatment on the expressions of miR-128/192/194.

The expression of two of the eight miRNAs were validated in a new set of samples using real-time quantitative PCR. We found that the expression of miR-375 was significantly decreased in response to $\mathrm{Pdx}-1$ induction (by $\sim 50 \%, \mathrm{p}<0.05$ ), whereas the miR-194 expression was significantly increased in response to IL- $1 \beta$ treatment (by $\sim 50 \%, \mathrm{p}<0.05$ ), see Figure 3. Furthermore, there was a tendency towards a decreased miR-194 expression in response to $\mathrm{Pdx}-1$ induction (by $\sim 50 \%, \mathrm{p}=0.05$ ). These expression changes confirmed the results from the array.

miRNA target prediction using negative correlation or ICA To assess the effect of the Pdx-1- and IL- $1 \beta$-mediated miRNA activity, mRNAs were profiled in the same set of samples as used for the miRNA arrays.
Initially, targets were predicted for each of the eight miRNAs using 6 mer seed matching. The total number of 6 mer seed matches for each miRNA is listed in Table 1. For identification of miRNA targets using negative correlation, correlation coefficients were calculated between each pair of miRNA and mRNA profiles and the mRNAs were ordered according to these correlation coefficients. Four of the eight miRNAs showed a significant enrichment of sequence predicted targets among mRNAs with correlation coefficients close to -1 ( $\mathrm{q}<$ 0.1 ), Table 1. Additional file 2 lists all calculated correlation coefficients.

Applying ICA on the mRNA expression data resulted in identification of five highly significant ICs (Bonferroni corrected $\mathrm{q}<0.0001$ ) with mixes shown in Figure 2B. See Additional file 2 for all loads, as well as the method section for further details on ICA. 


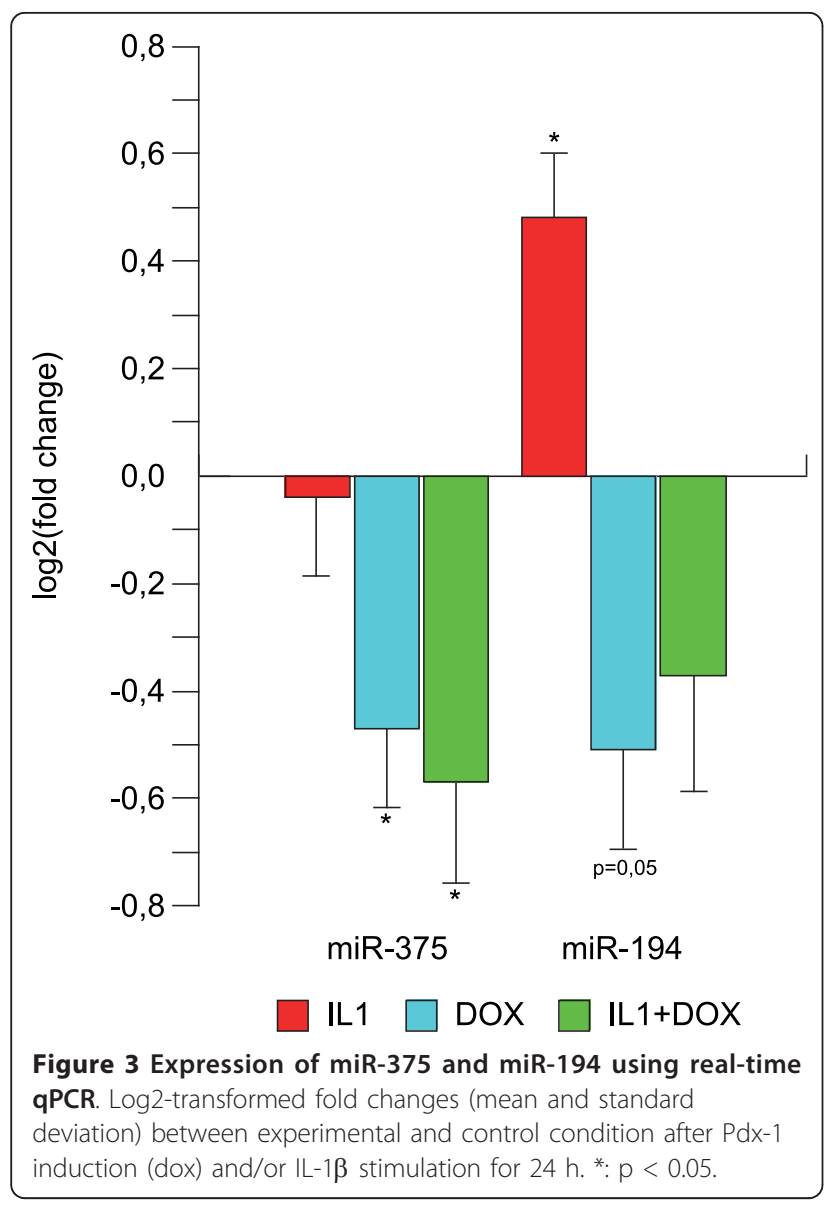

For each of the eight miRNAs, the loads in each IC were tested for enrichment of predicted targets with a 6 mer seed match. Seven of the eight miRNAs (including all four identified by negative correlation) showed a significant enrichment of sequence predicted targets in the IC loads ( $\mathrm{q}<0.07)$, Table 1 . Furthermore, we analyzed IC 1 for cooperativity between each pair of

Table 1 Target prediction using negative correlation or ICA (loads)

\begin{tabular}{clll}
\hline miRNA & \# 6mer matches & Correlation (q-value) & Load (q-value) \\
\hline miR-124 & 2215 & 0.0027 & 0.005 \\
\hline miR-128 & 2105 & 0.63 & 0.07 \\
\hline miR-192 & 1128 & 0.63 & 0.0007 \\
\hline miR-194 & 1110 & 0.0016 & 0.02 \\
\hline miR-204 & 1560 & 0.63 & 0.02 \\
\hline miR-375 & 1128 & 0.0027 & 0.04 \\
\hline miR-672 & 1060 & 0.87 & 0.5 \\
\hline miR-708 & 1675 & 0.023 & 0.0007 \\
\hline
\end{tabular}

The table shows the number of 6 mer seed matches in the array data for each of the eight miRNAs ( $2^{\text {nd }}$ column), the q-values (corrected $p$-values) for negative correlation ( $3^{\text {rd }}$ column) or load values ( $4^{\text {th }}$ column). The $q$-values in the $4^{\text {th }}$ column are the lowest q-value found among the five significant ICs.
miRNAs by testing the loads in IC 1 for enrichment of predicted targets with seed matches for both miRNAs (Additional file 3).

We found that the miRNA pairs miR-375/672, miR194/375, miR-192/375 and miR-124/194 had a significant regulatory effect (Bonferroni corrected $\mathrm{q}<0.05$ ) when testing for enrichment of their common targets in IC 1.

The miRNAs had small fold changes compared to the fold changes of the mRNAs. Therefore it was not possible to apply ICA on the miRNA data or on a combination of the mRNA and miRNA expression matrices. However, by forming a linear superposition of the ICs we identified the major contributions from the ICs on the miRNA expressions. Interestingly, all eight miRNAs could be represented (percent variance explained $>97 \%$ ) by a superposition of the five ICs, as shown in Figure 2C (also see Additional file 4). The majority of predicted miRNA targets were present in two or three of the ICs. For example, miR-124 primarily has targets in component 1 and 4, which both are Pdx-1 affected components. Another example is miR-192 and miR194 that both have targets in component 1, 2 and 4, which were affected by both Pdx-1 and IL-1 13 .

\section{Independent components have clear biological profiles}

To assess the biological relevance of the identified ICs, we tested the ICs for enrichment of genes known to be regulated by Pdx-1 or affected by IL- $1 \beta$ (Additional file 5). Mixes of IC 1 clearly correlated with the induction of $\mathrm{Pdx}-1$, and genes regulated by $\mathrm{Pdx}-1$ were overrepresented in this IC ( $\mathrm{p}<0.047$, data not shown). Interestingly, one group had low negative loads and another had high positive loads, indicating positive and negative regulation by $\mathrm{Pdx}-1$, respectively. Similarly, ICs 3 and 4 correlated with the presence or absence of IL$1 \beta$. Specifically, IC 3 was enriched for known IL-1 $\beta$ affected genes among genes with low negative load $(\mathrm{p}<$ 0.0021 , data not shown), whereas IC 4 was enriched for IL-1 $\beta$ affected genes with high positive load $(\mathrm{p}<0.024$, data not shown).

In a similar manner, we tested the ICs for enrichment of miRNA targets associated with metabolic or signalling pathways, as annotated by KEGG [38] or by the Molecular Signatures Database (MSigDB, http://www.broadinstitute.org). Specifically, we tested the genes with high positive or low negative loads in each IC for overrepresentation of annotated pathway genes.

When using KEGG terms only, 25 pathways were significantly enriched for miRNA targets in the five ICs $(\mathrm{q}<0.05)$. The most significant and diabetes-relevant pathways are shown in Figure 4 (all are listed in Additional file 6). Notable was the significance of genes with low loads in IC 3 belonging to the T1D pathway. IC 


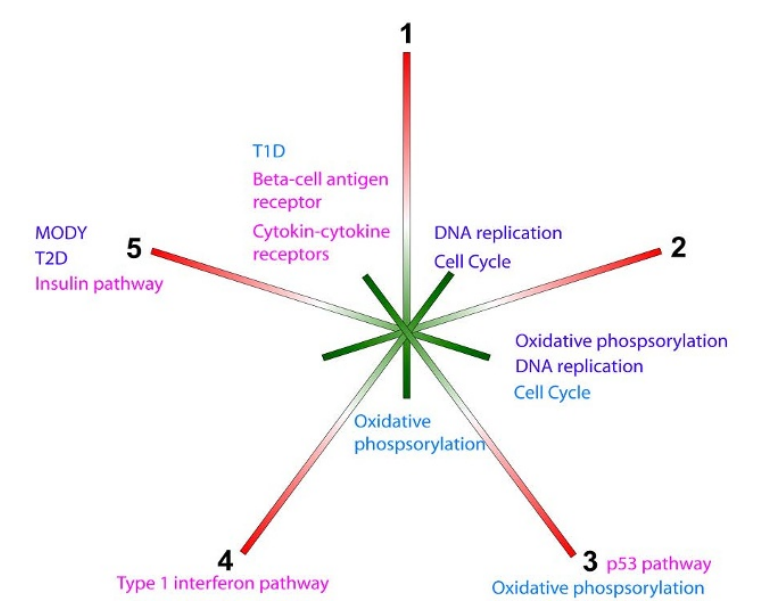

Figure 4 miRNA target-enriched pathways. Diagram showing a selection of the significant pathways for the miRNA targets in the five ICs when using KEGG annotations (blue text), MSigDB annotations (magenta text) or both annotations (purple text). The color of the bars indicate positive loads (red line) and negative loads (green line) of the miRNA targets in the ICs.

3 had a clear correlation with stimulation of IL-1 $\beta$. Furthermore, the pathways maturity onset diabetes of the young (MODY), type 2 diabetes (T2D) mellitus and oxidative phosphorylation were significant. The first two have an obvious relation to diabetes, and the oxidative phosphorylation pathway has been shown to be related to both type 1 and type 2 diabetes [39]. When using MSigDB annotations, 150 pathways were significantly enriched for miRNA targets belonging to the five ICs $(q<0.05)$. A selection of the pathways is shown in Figure 4 (all are listed in Additional file 6). Since KEGG is part of MSigDB it comes as no surprise that T2D, MODY and oxidative phosphorylation again showed up as significant. However, T1D did not show up as a significant pathway, probably due to correction of multiple testing, since MSigDB is a much larger repository. Also, dysregulation of genes involved in the p53 signaling pathway have been suggested to sensitize the cells to apoptotic stimuli $[32,40]$. In accordance with this, we find genes annotated with the p53-signalling pathway (using MSigDB) having significant low loads in IC 3.

\section{Identification of miRNA regulatory networks}

miRNAs and mRNAs can interact in regulatory networks. miRNAs can regulate mRNAs directly or indirectly through secondary factors. Furthermore, mRNA targets can act as transcription factors for miRNAs, thus forming a regulatory loop.

Using a combined bioinformatics approach involving seed matching, promoter analysis, text mining and ICA we identified miRNA regulatory networks (Figure 5). Using the miRNA database http://www.miRbase.org, we

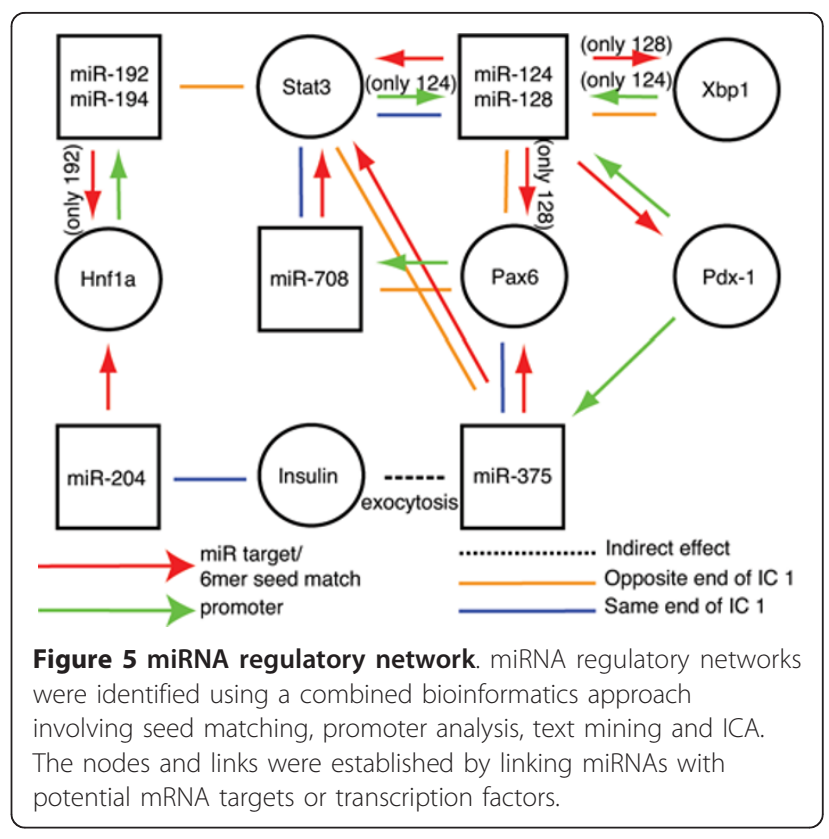

found STAT3 binding sites in the promoter of the human miR-124 gene. This interaction was supported by ICA that showed that miR-124 and Stat3 both have negative contribution from IC 1 (Additional file 2 and Figure $2 \mathrm{C}$ ) correlating with an up-regulation in response to Pdx-1 induction. Interestingly, the 3'UTR of Stat3 has seed matches for miR-124 (TargetScan and 6mer seed matching), indicating a potential feedback loop.

Furthermore, we found positive and negative contribution from IC 1 for Pax6 and miR-124/128, respectively (Additional file 2 and Figure 2C), meaning they have reciprocal expressions (down-regulation of Pax6 and upregulation of miR-124/128) in response to $\mathrm{Pdx}-1$ induction. Interestingly, the 3'UTR of Pax6 contains a 6mer seed match for miR-128 indicating potential repression of Pax6 by miR-128.

\section{Validation in human pancreatic islets and $\beta$-cell studies}

To verify the identified miRNA targets affected by Pdx1 and/or cytokine stimulation, we used mRNA expression profiling data from studies on human pancreatic islets and rat $\beta$-cell lines and tested whether the data were enriched for ICA-identified targets of the eight miRNAs.

Using mRNA profiling data from untreated and cytokine treated human pancreatic islet preparations (unpublished data) we tested the cytokine-induced fold changes for enrichment of predicted miRNA targets. Interestingly, we found that predicted targets of miR$128 / 192 / 194 / 204 / 375$ were significantly up-regulated in response to cytokine treatment as compared to non$\operatorname{targets}(\mathrm{q}=0.007, \mathrm{q}=0.004, \mathrm{q}=0.033, \mathrm{q}=0.001$ and 
$q=0.05$, respectively). The results suggest that the five miRNAs may play a role in human pancreatic islets as well.

Additionally, we performed similar analysis of publicly available data from two previous studies on $\beta$-cells. Cardozo et al. [41] performed mRNA profiling on $\beta$-cells from 10 weeks old male Wistar rats, un-stimulated or stimulated with cytokines (IL-1 $\beta$ and/or IFN $\gamma$ ). Using their mRNA data, we found that targets of miR192 were significantly down-regulated in response to IL$1 \beta$ stimulation when comparing to non-targets $(\mathrm{q}=$ 0.02). Kutlu et al. [42] performed mRNA profiling on insulin producing INS-1E cells, untreated or exposed to a combination of IL- $1 \beta$ and IFN $\gamma$. In their data set, we found that the expressions of miR-128 targets were significant increased in response to cytokine exposure when comparing to non-targets $(\mathrm{q}=0.002)$.

\section{Discussion}

The motivation for applying a more advanced method like ICA for miRNA target prediction than negative correlation was that surprisingly few mRNAs have clear negative correlations with their targeting miRNAs. This is probably due to the mRNA profiles being influenced by a number of factors e.g. miRNA regulation, transcription factor binding and site accessibility. Here, ICA was used as an attempt to filter the factors influencing mRNA expression. Decomposition of microarray data using ICA has been shown to outperform other linear data representations, such as PCA [16-19]. Several target prediction methods have incorporated miRNA and mRNA profiling data. However, none of them uses ICA.

The miRNA array profiling identified eight miRNAs with differential expression in a type 1 diabetes model. Performing ICA on the mRNA expressions from the same samples resulted in five ICs that correlated with the experimental conditions studied. Comparing the two target prediction methods indicated that ICA was better at capturing miRNA activity than negative correlation. Seven miRNAs showed a significant enrichment of sequence-predicted targets when using ICA, as compared to only four by use of negative correlation. Interestingly, the ICs were enriched for miRNA targets with functional roles in diabetes-relevant pathways e.g. the pathways T1D, T2D, MODY, oxidative phosphorylation, insulin, cytokine-cytokine receptors and type 1 interferon. This supports that the eight miRNAs are implicated in disease mechanisms in diabetes. Additionally, targets of five of the eight miRNAs were significantly regulated by cytokines in models of $\beta$-cell destruction e.g. in human islets.

miRNAs fine tune the expression of genes in a combinatorial manner, meaning that several miRNAs can target the same mRNA transcript [43]. Furthermore, a cluster of co-expressed miRNAs can regulate functionally related genes [44]. In this study, we observe small expression changes in the miRNAs. However, even minute changes in miRNA expressions might have impact on mRNA expression, and miRNAs acting in a cooperative manner can most likely induce biologically relevant expression changes in their targets. ICA can uncover these more complex interactions. Interestingly, it was recently suggested that cooperativity could be incorporated for prediction of target interactions between different miRNAs [1]. For the eight miRNAs we identified, there is a significant overlap in the mRNAs they target. We have incorporated cooperativity between miRNAs pairwise and identified four miRNA pairs (miR-375/672, miR-194/375, miR-192/375, miR$124 / 194$ ) that had a significant co-regulatory effect on their common targets in IC 1.

Of the eight significant miRNAs, miR-124 and miR375 have previously been identified in $\beta$-cells $[33,35,45,46]$. Further, the expression of miR-204 has been shown to be induced in insulinomas, where its expression correlated with insulin expression [47].

That miR-375 was significantly regulated strengthens our model since a previous study observed interaction between Pdx-1 (and NeuroD1) and the miR-375 locus [32]. However, no Pdx-1 consensus binding sites were identified, but binding elements for other transcription factors have been identified in the miR-375 locus [48]. The decreased miR-375 expression could, at least in part, be mediated through NeuroD1, since we observed a decreased NeuroD1 expression in response to Pdx1 induction. Additionally, the decreased miR-375 expression is in agreement with miR-375 having a higher expression level in non- $\beta$-cells than in $\beta$-cells [35]. This is also supported by our findings in $\alpha$-cells versus $\beta$-cells (Additional file 7). Interestingly, both miR375 and Pax6, a key factor in $\alpha$-cell development, had negative loads in IC 1, i.e. both were down-regulated in response to Pdx-1. The decreased miR-375 expression is also in compliance with the function of miR-375 as a negative regulator of insulin exocytosis [45], since it correlates with the need for an increased insulin secretion in the mature $\beta$-cell phenotype. Similarly, miR-124 has been shown to modulate insulin secretion by targeting Foxa2 [33]. miR-194 is highly expressed in liver and in intestinal epithelial cells, where it is under regulation by $\mathrm{Hnf} 1 \alpha[49,50]$. Interestingly, $\mathrm{Hnf} 1 \alpha$ is required for proper $\beta$-cell function and mutations in this gene cause MODY [23]. miR-192 is also expressed in the liver and is in cluster with miR-194, suggesting co-regulation $[49,50]$. Furthermore, miR-128 has been shown to induce apoptosis in kidney cells through interaction with Bax [51]. So far, miR-672 and miR-708 have not been examined in $\beta$-cells. The likely involvement of miR-128/192/194/204/708 in $\beta$-cell regulatory networks 
(Figure 5) and the high expression in $\beta$-cells compared to $\alpha$-cells for miR-672/204 (Additional file 7) make them interesting candidates for further studies.

We have used ICA for bioinformatics investigation of the functional roles of the miRNAs and their targets. ICA in combination with pathway analysis indicates that the eight miRNAs, through their mRNA targets, are implicated in several diabetes relevant pathways.

The transcriptional changes mediated by miRNAs on their targets may not be entirely explained by direct repression but may also reflect indirect mechanisms such as activation by feedback and feed-forward transcriptional loops within regulatory networks $[52,53]$. miRNAs can be important players in these networks. By use of a combined bioinformatics approach we identified miRNA regulatory networks. The results suggest connections between seven of the eight miRNAs through interactions with key pancreatic transcription factors, cytokine signalling molecules and insulin (Figure 5).

\section{Conclusions}

Using ICA, we have developed a novel miRNA target prediction method that incorporates seed matching and expression profiling. We believe that the method has advantages compared to simple negative correlation. Additionally, ICA in combination with bioinformatics approaches such as pathway analysis constitutes a means of prioritizing between the predicted miRNA targets for further investigations. To the best of our knowledge this is the first study that uses ICA for miRNA target prediction. Interestingly, applying the method on a model of type 1 diabetes resulted in identification of eight miRNAs that appear to directly or indirectly affect pathways of relevance to disease mechanisms in diabetes.

\section{Methods}

\section{Cell cultures}

INSr $\alpha \beta, \beta$ TC- 3 and $\alpha$ TC- 1 cells were cultured in complete RPMI-1640 medium (RPMI-1640 with Glutamax (Gibco BRL, Paisley, Scotland, UK), $100 \mathrm{U} / \mathrm{ml}$ penicillin and $100 \mu \mathrm{g} / \mathrm{ml}$ streptomycin) supplemented with $10 \%$ FBS. $50 \mu \mathrm{M} \beta$-mercaptoethanol was additionally added to the INSr $\alpha \beta$ cells. For the INSr $\alpha \beta$ cells, TeT system approved FBS (CLONTECH, Palo Alto, CA) was used, and the medium was supplemented with $150 \mu \mathrm{g} / \mathrm{ml}$ geneticin and $100 \mu \mathrm{g} / \mathrm{ml}$ hygromycin to maintain a pure culture of cells expressing the Pdx-1 plasmid construct. The INSr $\alpha \beta$ cells were treated with doxycycline (500 ng/ml; Sigma Aldrich, Saint Louis, MO, USA) for $24 \mathrm{~h}$ to allow for Pdx-1 expression after which the cells were cultured in the presence or absence of human recombinant IL-1 $\beta$ ( $40 \mathrm{pg} / \mathrm{ml}$; BD Pharmingen, San Diego, CA) for additionally $2 \mathrm{~h}$ or $24 \mathrm{~h}$.

\section{Human pancreatic islet preparations}

Human islet preparations $(n=4)$ provided through the Juvenile Diabetes Research Foundation (JDRF) Islet Distribution Program (JDRF award 6-2005-1178) by Islet Cell Resource Centres in Milan (Italy) and Lille (France) were treated for 48 hours with IL-1 $\beta$ ( $1 \mathrm{ng} / \mathrm{ul})$ or a combination of IL-1 $\beta$ ( $1 \mathrm{ng} / \mathrm{ul})$, IFN $\gamma(20 \mathrm{ng} / \mathrm{ul})$ and TNF $\alpha$ (8 ng/ul).

\section{RNA extraction and RT-qPCR}

Total RNA was extracted using TRIzol reagent (Invitrogen, Carlsbad, CA) according to the manufacturer's instructions. cDNA was prepared using miScript reverse transcription kit (Qiagen, Hilden, Germany), TaqMan microRNA reverse transcription reagents (Applied Biosystems, Foster City, CA) or TaqMan reverse transcription reagents (Applied Biosystems), as described by the manufacturer.

In INSr $\alpha \beta$ cells $(n=5)$ the miRNA expression levels were analysed by use of miScript Primer Assays (Qiagen), whereas in $\beta$ TC- $3(n=5)$ and $\alpha$ TC- $1(n=5)$ cells miRNA expressions were analysed using TaqMan microRNA assays (Applied Biosystems). Gene expression levels were analysed using TaqMan gene expression assays (Applied Biosystems). All samples were analyzed in duplicates on a ABI 7900HT system (Applied Biosystems). Data were evaluated using the $2^{-\Delta \Delta \mathrm{Ct}}$ method [54], normalizing miRNA/gene expressions to an endogenous control, and subsequently comparing each miRNA/gene in treated vs. un-stimulated cells. miRNAs were normalized to let-7c, whereas genes were normalized to Ppia. The coefficient of variation (CV) for miScript assays ranged from 0.018 to 0.077 .

\section{Western blotting}

The INSr $\alpha \beta$ cells $(n=4)$ were washed with PBS and lysed. The protein concentrations of whole cell protein lysates were measured with Bio-Rad Protein Assay. Samples were boiled before loading on a NuPage gel $(10 \%$ BisTris gels, Invitrogen) placed in an X-Cell Surelock system (Invitrogen). Proteins were separated and then transferred to a pre-soaked nitrocellulose membrane (Invitrogen), which was blocked in milk for one hour and then washed. The membrane was incubated in a $5 \mathrm{ml}$ milk solution containing primary antibody, either monoclonal Pdx-1 mouse antibody [37] overnight or monoclonal mouse $\beta$-actin antibody (Abcam, Cambridge, MA; \#ab6276) for one hour. Horseradish peroxidase conjugated anti-mouse antibodies (Cell signalling, \#7076) were used as secondary antibodies. The membrane was incubated in LumiGlo (Cell Signialing Technology, Danvers, MA) solution and visualized using the LAS2000 system (Fujifilm Europa GmbH, Dusseldorf, Germany). 


\section{Profiling and preprocessing of array data}

The mRNA expressions from INSr $\alpha \beta$ cells $(n=4)$ were profiled on Affymetrix Rat Genome 2302.0 arrays. For each probeset in each sample, log2-ratios were calculated between the expression of that probeset and the average of the control samples.

The miRNA expressions from INSr $\alpha \beta$ cells $(n=3)$ were profiled on Exiqon miRCURY LNA microRNA Array v.11.0 arrays and median normalized. The control samples $(\mathrm{n}=3)$ were pooled and used as a common reference on all arrays, and $\log 2$-ratios were calculated for each sample against this reference.

The mRNA expressions from human islet preparations $(\mathrm{n}=4)$ were analyzed on Affymetrix Human Genome U133 Plus 2.0 arrays. The $\beta$-cell data from Refs. [41] and [42] were downloaded from T1DBase http://www. t1dbase.org. These mRNA expressions had been profiled using the Affymetrix Rat Genome U34A array.

All CEL-files were preprocessed using the RMA package [55] in Bioconductor [56] with remapped Ensembl build 50 gene probesets [57].

For mRNA data from human islets and published $\beta$-cell studies, foldchanges $\log 2$ (cytokine, $24 \mathrm{~h}$ )/ (untreated, $24 \mathrm{~h}$ ) were calculated for the average of the replicate samples in each condition.

\section{Sequence based miRNA target prediction}

For 7764 out of the 9953 Ensembl gene probesets analyzed, 3'UTR sequences could be downloaded from Ensembl through Biomart [58]. Mature sequences of significantly changed miRNAs were downloaded from miRbase [59] and the 6mer seed matches extracted. Ensembl gene probesets with a given miRNA 6mer seed match in their 3'UTR were identified as targets of that miRNA.

\section{Independent component analysis}

Mathematically, $T$ transcriptional programs are active in the cells under study, each of which is a (column) vector $\vec{C}_{t}, t=1, . ., T$, representing $G_{t}$ gene inductions or repressions. We measure all $G$ induced or repressed genes in $S$ samples.

Each of these programs can be identified by a simple linear decomposition of the $G \times S$ expression matrix E,

$$
\mathrm{E}=\mathrm{CM} \text { or } \mathrm{E}_{\mathrm{gs}}=\sum_{t=1}^{T} C_{g t} \mathrm{M}_{t s} \text { or } \vec{E}_{s}=\sum_{t=1}^{T} C_{t} M_{t s}
$$

where $C$ is the $G \times T$ matrix containing the transcriptional programs, in coefficient vector notation, $C=\left(\vec{C}_{1}, \vec{C}_{2}, \ldots, \vec{C}_{T}\right)$, likewise $E=\left(\vec{E}_{1}, \vec{E}_{2}, \ldots, \vec{E}_{S}\right)$ and $M$ is the $T \times S$ matrix giving the linear mixing of each program in each sample. Two-way clustering of correlated genes and samples into non-overlapping sets was represented by component vectors $\vec{C}_{t}$ with discrepant sets of non-vanishing entries

$$
C_{g t} \neq 0 \Leftrightarrow C_{g j} \approx 0 \forall j \neq t .
$$

ICA extracts $\mathrm{n}=\min \{G, S\}$ independent components. Since the number of samples was much lower than the number of genes, we assumed $\mathrm{n}=S$. The number of interesting transcriptional programs $T$ may be equal to, or lower than $S$. A common practice is to project $\mathrm{E}$ onto it's first $O$ principal component directions, and then extract $O$ independent components in this subspace. This approach has several important drawbacks. First, the number of interesting transcriptional programs is not known beforehand, and one therefore needs to guess or resort to a recursive trial-and-error approach to choose an $O$ that allows all $T$ transcriptional programs to be extracted. Second, it is not obvious that a projection onto $O<S$ principal component directions preserves all $T$ transcriptional programs. It is possible to construct examples where two clusters in the data are only well separated in the subspace of the first and last principal components [60]. Clustering in a subspace spanned by principal components generally degrades cluster quality [61]. Finally, even when a projection onto $T$ principal components allows all the $T$ interesting transcriptional programs to be extracted by ICA, a way to explore and rank these independent components to clarify their significance is still needed. In the present study, the independent components were ranked according to their accordance with the experimental conditions.

The fastICA algorithm [62,63] in R http://www.R-project.org was used to estimate the component matrix and mixing matrix, by numeric maximization of the negentropy of the independent components. The negentropy was approximated by $J(y)=(\langle G(y)\rangle-\langle G(v)\rangle)^{2}$, where $y$ is the distribution of loads, $\mathrm{v}$ is a random variable distributed as $\boldsymbol{\aleph}(0,1)$, and the contrast function $G(u)$ was here defined as $G(u)=-\exp \left(\frac{-u^{2}}{2}\right)$. By convention, the loads in each component have mean 0 and variance 1 .

Assuming that the individual contributions on mRNA expression are linearly separable and reasonable independent of each other, ICA can separate the various contributions making it possible to detect pathways regulated by the various miRNAs.

ICs with mixes, i.e. rows in $M$ showing significant changes between the experimental conditions and/or time points, are identified by calculating F-statistics and requiring Bonferroni corrected p-values less than 0.0001 . 


\section{Statistics}

Overall expression data were analysed using one-way analysis of variance (ANOVA; F-statistics). The eight significant miRNAs were selected based on an overall q-value $<0.1$ (Additional file 8). Comparison between groups was done using t-test. Correction for multiple testing was performed either by applying a Bonferroni or a false discovery rate (fdr) correction to the p-values (q-value). A Bonferroni correction was chosen when few tests were performed, as was the case for the finding of significant ICs and miRNAs. When a larger number of tests were performed a fdr correction was applied.

The loads in each IC are considered non-Gaussian distributed. Loads/fold changes for genes that had seed match for the miRNAs or were assigned to a specific biological pathway were analysed using Wilcoxon rank sum test. For analysis of negative correlation, Pearson correlation coefficients were calculated between each pair of miRNA and mRNA profiles. The mRNAs were then ordered according to these correlation coefficients. P-values $<0.05$ were considered significant.

\section{Additional material}

\section{Additional file 1: Expressions of insulin and $\mathrm{Pdx}-1$ dependent genes}

There are three experimental conditions: Pdx-1 induction (dox treatment), $\mathrm{L}-1 \beta$ treatment and time (samples are taken $2 \mathrm{~h}$ and $24 \mathrm{~h}$ after treatment). Log2-transformed fold changes (mean and standard deviation) between experimental and control conditions. ${ }^{*}: 0.05>\mathrm{q}>$ $0.01,{ }^{* *}: 0.01>q>0.001,{ }^{* * *}: 0.001>q>0$.

\section{Additional file 2: miRNA target prediction based on mRNA} expression data. For each Ensembl annotated gene on the Affymetrix array is given the gene symbol, gene description, loads in the five ICs, coefficients for correlation with the eight miRNA expression profiles and 6 mer seed match with the eight miRNAs. We assumed that loads were significant if they had an absolute load greater than 2. For seed match, $1 / 0$ denotes $\geq 1$ seed matches and no seed match.

Additional file 3: Cooperativity between miRNAs in IC 1. The $p$ values (uncorrected) and q-values (corrected) for pairwise miRNA cooperativity in IC 1 are shown. 1/-1 denotes whether the individual miRNA has positive or negative loads in IC 1.

Additional file 4: Coefficients for the linear superposition of the ICs giving the miRNA expression profiles. R2 is the coefficient of determination.

Additional file 5: Genes regulated by Pdx-1 and/or IL-1 $\beta$. 1/0 denotes regulation and no regulation based on text mining.

Additional file 6: Pathways significantly enriched for genes with high positive or low negative loads in the ICs. Sheet 1 shows KEGG pathways whereas sheet 2 shows MSigDB pathways. Only pathways with q-values $<0.05$ ( $f d r$ corrected p-values) are highlighted.

Additional file 7: Expression of the eight miRNAs in $\alpha$ - versus $\beta$ cells. Ratio of basal $\triangle C T$ values for $\alpha T C 1$ versus $\beta T C 3$ cells. The ratio is found for un-stimulated cells. Bars are standard deviations and asterisks denote: ${ }^{* *}: 0.01>q>0.001,{ }^{* *}: 0.001>q>0$.

Additional file 8: Overall q-values for the miRNA expressions. FWER: family-wise error rate.

\section{List of abbreviations}

3'UTR: untranslated region in the $3^{\prime}$ end of the mRNA; dox: doxycycline; FBS: fetal bovine serum; IC: independent component; ICA: independent component analysis; IFNy: interferon gamma; IL-1 $\mathbf{\beta}$ : interleukin 1 beta; $\mathbf{m i R /}$ miRNA: microRNA; MODY: maturity onset diabetes of the young; $\mathbf{m R N A}$ : messenger RNA; PBS: phosphate buffered saline; PCA: principal component analysis; RT-qPCR: reverse transcription - quantitative polymerase chain reaction; T1D: type 1 diabetes; T2D: type 2 diabetes; TNFa: tumor necrosis factor alpha.

\section{Acknowledgements and Funding}

This work was supported by grants from The Danish Diabetes association, EFSD (European Foundation for the Study of Diabetes) and the Danish Council for Strategic Research "Center for non-coding RNA in Technology and Health" (09-067036/DSF). The work of PHH was supported by a grant from the Lundbeck Foundation. We are grateful to Drs Haiyan Wang and Claes B. Wollheim University of Geneva for kindly providing the INSr $\alpha \beta$ cell line. We thank Bodil Bosmann Jørgensen, Hagedorn Research Institute, for technical assistance in the lab, and additionally we thank Jacob Hald also from Hagedorn Research Institute for providing the PDX-1 antibody. We thank Jens Højriis Nielsen and Maiken Rosenstierne at University of Copenhagen for providing access to array facilities and expert guidance in miRNA array methods. Drs. AE Karlsen, M Kruhöffer and T Ørntoft are acknowledged for taking part in the design and carrying out the mRNA Affymetrix array experiments.

\section{Author details}

${ }^{1}$ Glostrup Research Institute, Glostrup University Hospital, DK-2600 Glostrup, Denmark. ${ }^{2}$ Hagedorn Research Institute, Niels Steensensvej 6, DK2820 Gentofte, Denmark. ${ }^{3}$ Center for Models of Life, University of Copenhagen, Blegdamsvej 17, DK-2100 Copenhagen, Denmark. ${ }^{4}$ Center for Biological Sequence Analysis, Department of Systems Biology, Technical University of Denmark, DK-2800 Lyngby, Denmark. ${ }^{5}$ Steno Diabetes Center, Niels Steensensvej 2, DK-2820 Gentofte, Denmark. ${ }^{6}$ University of Lund, CRC, Skåne University Hospital, SE-20502 Malmoe, Sweden. ${ }^{7}$ Department of Molecular Biomedicine, LEO Pharma A/S, Industriparken 55, DK-2750 Ballerup, Denmark.

\section{Authors' contributions}

FP and CHBB designed the study. The experimental work was performed by $\mathrm{CHBB}, \mathrm{TF}$ and TG. The analyses and the manuscript draft were conducted by $L P, C H B B$ and TF. ICA and microarray data analysis were conducted by LP and $\mathrm{PHH}$. FP edited the paper and prepared it for final review. All authors read and approved the final manuscript and have no conflicting interest in the study.

Received: 9 August 2010 Accepted: 4 February 2011

Published: 4 February 2011

\section{References}

1. Saito T, Sætrom P: MicroRNAs - targeting and target prediction. NeW Biotechnology 2010, 27(3):243-249.

2. Sood P, Krek A, Zavolan M, Macino G, Rajewsky N: Cell-type-specific signatures of microRNAs on target mRNA expression. PNAS 2006, 103(8):2746-2751.

3. Huang JC, Babak T, Corson TW, Chua G, Khan S, Gallie BL, Hughes TR, Blencowe BJ, Frey BJ, Morris QD: Using expression profiling data to identify human microRNA targets. Nat Methods 2007, 4(12):1045-1049.

4. Dongen Sv, Abreu-Goodger C, Enright AJ: Detecting microRNA binding and siRNA off-target effects from expression data. Nat Methods 2008, 5(12):1023-1025

5. Cheng C, Li LM: Inferring microRNA activities by combining gene expression with microRNA target prediction. PLOS ONE 2008, 3(4):e1989.

6. Perou CM, Sørlie T, Eisen MB, Rijn Mvd, Jeffrey SS, Rees CA, Pollack JR, Ross DT, Johnsen $H$, Akslen LA, et al: Molecular portraits of human breast tumours. Nature 2000, 406(6797):747-752.

7. Arbeitman MN, Furlong EEM, Imam F, Johnson E, Null BH, Baker BS, Krasnow MA, Scott MP, Davis RW, White KP: Gene expression during the life cycle of Drosophila melanogaster. Science 2002, 297(5590):2270-2275. 
8. Schmid M, Davison TS, Henz SR, Pape UJ, Demar M, Vingron M, Schölkopf B, Weigel D, Lohmann JU: A gene expression map of Arabidopsis thaliana development. Nat Genet 2005, 37(5):501-506.

9. Ohlsson Teague EMC, Van der Hoek KH, Van der Hoek MB, Perry N, Wagaarachchi P, Robertson SA, Print CG, Hull LM: MicroRNA-Regulated Pathways Associated with Endometriosis. Mol Endocrinol 2009, 23(2):265-275

10. Comon P: Independent component analysis, A new concept? Signal Processing 1994, 36:287-314.

11. Martoglio AM, Miskin JW, Smith SK, MacKay DJC: A decomposition model to track gene expression signatures: preview on observer-independent classification of ovarian cancer. Bioinformatics 2002, 18(12):1617-1624.

12. Chiappetta P, Roubaud MC, Torrésani B: Blind source separation and the analysis of microarray data. J Comput Biol 2004, 11(6):1090-1109.

13. Saidi SA, Holland CM, Kreil DP, MacKay DJC, Charnock-Jones DS, Print CG, Smith SK: Independent component analysis of microarray data in the study of endometrial cancer. Oncogene 2004, 23(39):6677-6683.

14. Capobianco E: Mining time-dependent gene features. J Bioinform Comput Biol 2005, 3(5):1191-1205.

15. Lutter D, Ugocsai P, Grandl M, Orso E, Theis F, Lang E, Schmitz G: Analyzing M-CSF dependent monocyte/macrophage differentiation: expression modes and meta-modes derived from an independent component analysis. BMC Bioinformatics 2008, 9(1):100.

16. Liebermeister $\mathrm{W}$ : Linear modes of gene expression determined by independent component analysis. Bioinformatics 2002, 18(1):51-60.

17. Lee SI, Batzoglou S: Application of independent component analysis to microarrays. Genome Biol 2003, 4(11):R76.

18. Carpentier AS, Riva A, Tisseur P, Didier G, Hénaut A: The operons, a criterion to compare the reliability of transcriptome analysis tools: ICA is more reliable than ANOVA, PLS and PCA. Comput Biol Chem 2004, 28(1):3-10.

19. Teschendorff AE, Journée M, Absil PA, Sepulchre R, Caldas C: Elucidating the altered transcriptional programs in breast cancer using independent component analysis. PLoS Comput Biol 2007, 3(8):e161.

20. Frigyesi A, Veerla S, Lindgren D, Höglund M: Independent component analysis reveals new and biologically significant structures in micro array data. BMC Bioinformatics 2006, 7:290.

21. Eizirik DL, Flodstrom $M$, Karlsen $A E$, Welsh $N$ : The harmony of the spheres: inducible nitric oxide synthase and related genes in pancreatic beta cells. Diabetologia 1996, 39(8):875-890.

22. Lynn FC, Skewes-Cox P, Kosaka Y, McManus MT, Harfe BD, German MS: MicroRNA expression is required for pancreatic islet cell genesis in the mouse. Diabetes 2007, 56(12):2938-2945.

23. Bernardo AS, Hay CW, Docherty K: Pancreatic transcription factors and their role in the birth, life and survival of the pancreatic beta cell. Mol Cell Endocrinol 2008, 294(1-2):1-9.

24. Jonsson J, Carlsson L, Edlund T, Edlund H: Insulin-promoter-factor 1 is required for pancreas development in mice. Nature 1994, 371(6498):606-609.

25. Offield MF, Jetton TL, Labosky PA, Ray M, Stein RW, Magnuson MA Hogan BL, Wright CV: PDX-1 is required for pancreatic outgrowth and differentiation of the rostral duodenum. Development 1996, 122(3):983-995.

26. Stoffers DA, Zinkin NT, Stanojevic V, Clarke WL, Habener JF: Pancreatic agenesis attributable to a single nucleotide deletion in the human IPF1 gene coding sequence. Nat Genet 1997, 15(1):106-110.

27. Ohlsson H, Karlsson K, Edlund T: IPF1, a homeodomain-containing transactivator of the insulin gene. EMBO J 1993, 12(11):4251-4259.

28. Wang H, Maechler P, Ritz-Laser B, Hagenfeldt KA, Ishihara H, Philippe J, Wollheim CB: Pdx1 level defines pancreatic gene expression pattern and cell lineage differentiation. J Biol Chem 2001, 267(27):25279-25286.

29. Nielsen $K$, Karlsen AE, Deckert M, Madsen OD, Serup P, Mandrup-Poulsen T, Nerup J: Beta-cell maturation leads to in vitro sensitivity to cytotoxins. Diabetes 1999, 48(12):2324-2332.

30. Nielsen K, Kruhøffer M, Orntoft T, Sparre T, Wang H, Wollheim C, Jørgensen MC, Nerup J, Karlsen AE: Gene expression profiles during beta cell maturation and after IL-1beta exposure reveal important roles of Pdx-1 and Nkx6.1 for IL-1 beta sensitivity. Diabetologia 2004, 47(12):2185-2199.

31. Nielsen $K$, Sparre T, Larsen MR, Nielsen M, Fey SJ, Mose Larsen P, Roepstorff P, Nerup J, Karlsen AE: Protein expression changes in a cell system of beta-cell maturation reflect an acquired sensitivity to IL-1beta. Diabetologia 2004, 47(1):62-74.

32. Keller DM, McWeeney S, Arsenlis A, Drouin J, Wright CV, Wang H, Wollheim CB, White $\mathrm{P}$, Kaestner $\mathrm{KH}$, Goodman $\mathrm{RH}$ : Characterization of pancreatic transcription factor $\mathrm{Pdx}-1$ binding sites using promoter microarray and serial analysis of chromatin occupancy. J Biol Chem 2007, 282(44):32084-32092

33. Baroukh N, Ravier MA, Loder MK, Hill EV, Bounacer A, Scharfmann R, Rutter GA, Van Obberghen E: MicroRNA-124a regulates Foxa2 expression and intracellular signaling in pancreatic beta-cell lines. J Biol Chem 2007, 282(27):19575-19588

34. Joglekar MV, Parekh VS, Hardikar AA: New pancreas from old: microregulators of pancreas regeneration. Trends Endocrinol Metab 2007, 18(10):393-400.

35. Joglekar MV, Joglekar VM, Hardikar AA: Expression of islet-specific microRNAs during human pancreatic development. Gene Expr Patterns 2009, 9(2):109-113

36. Correa-Medina M, Bravo-Egana V, Rosero S, Ricordi $C$, Edlund $H$, Diez J, Pastori RL: MicroRNA miR-7 is preferentially expressed in endocrine cells of the developing and adult human pancreas. Gene Expr Patterns 2009, 9(4):193-199.

37. Galbo T, Pedersen I, Floyel T, Bang-Berthelsen C, Serup P, Madsen O, Hald J: Novel monoclonal antibodies against $\mathrm{Pdx} 1$ reveal feedback regulation of Pdx1 protein levels. European Journal of Histochemistry 2010, 54:e19.

38. Kanehisa M, Goto S, Furumichi M, Tanabe M, Hirakawa M: KEGG for representation and analysis of molecular networks involving diseases and drugs. Nucleic Acids Res 2010, , 38 Database: D355-360.

39. Mootha VK, Lindgren CM, Eriksson KF, Subramanian A, Sihag S, Lehar J, Puigserver P, Carlsson E, Ridderstrale M, Laurila E, et al: PGC-1[alpha]responsive genes involved in oxidative phosphorylation are coordinately downregulated in human diabetes. Nat Genet 2003, 34(3):267-273.

40. Gurzov EN, Germano CM, Cunha DA, Ortis F, Vanderwinden JM, Marchetti $P$, Zhang L, Eizirik DL: p53 up-regulated modulator of apoptosis (PUMA) activation contributes to pancreatic beta-cell apoptosis induced by proinflammatory cytokines and endoplasmic reticulum stress. $J$ Biol Chem 2010, 285(26):19910-19920.

41. Cardozo AK, Kruhoffer M, Leeman R, Orntoft T, Eizirik DL: Identification of novel cytokine-induced genes in pancreatic beta-cells by high-density oligonucleotide arrays. Diabetes 2001, 50(5):909-920.

42. Kutlu B, Cardozo AK, Darville MI, Kruhoffer M, Magnusson N, Orntoft T, Eizirik DL: Discovery of gene networks regulating cytokine-induced dysfunction and apoptosis in insulin-producing INS-1 cells. Diabetes 2003, 52(11):2701-2719.

43. Krek A, Grün D, Poy MN, Wolf R, Rosenberg L, Epstein EJ, MacMenamin P, Piedade Id, Gunsalus KC, Stoffel M, et al: Combinatorial microRNA target predictions. Nat Genet 2005, 37(5):495-500.

44. Dews M, Homayouni A, Yu D, Murphy D, Sevignani C, Wentzel E, Furth EE, Lee WM, Enders GH, Mendell JT, et al: Augmentation of tumor angiogenesis by a Myc-activated microRNA cluster. Nat Genet 2006, 38(9):1060-1065.

45. Poy MN, Eliasson L, Krutzfeldt J, Kuwajima S, Ma X, Macdonald PE, Pfeffer S, Tuschl T, Rajewsky N, Rorsman P, et al: A pancreatic islet-specific microRNA regulates insulin secretion. Nature 2004, 432(7014):226-230.

46. Poy MN, Hausser J, Trajkovski M, Braun M, Collins S, Rorsman P, Zavolan M, Stoffel M: miR-375 maintains normal pancreatic alpha- and beta-cell mass. Proc Natl Acad Sci USA 2009, 106(14):5813-5818.

47. Roldo C, Missiaglia E, Hagan JP, Falconi M, Capelli P, Bersani S, Calin GA, Volinia S, Liu CG, Scarpa A, et al: MicroRNA expression abnormalities in pancreatic endocrine and acinar tumors are associated with distinctive pathologic features and clinical behavior. J Clin Oncol 2006, 24(29):4677-4684.

48. Avnit-Sagi T, Kantorovich L, Kredo-Russo S, Hornstein E, Walker MD: The promoter of the pri-miR-375 gene directs expression selectively to the endocrine pancreas. PLoS One 2009, 4(4):e5033.

49. Tang $X$, Gal J, Zhuang $X$, Wang W, Zhu H, Tang G: A simple array platform for microRNA analysis and its application in mouse tissues. RNA 2007 13(10):1803-1822.

50. Hino K, Tsuchiya K, Fukao T, Kiga K, Okamoto R, Kanai T, Watanabe M: Inducible expression of microRNA-194 is regulated by HNF-1alpha during intestinal epithelial cell differentiation. RNA 2008, 14(7):1433-1442 
51. Adlakha Y, Saini N: MicroRNA-128 downregulates Bax and induces apoptosis in human embryonic kidney cells. Cellular and Molecular Life Sciences 2010, 1-14.

52. Chen K, Rajewsky N: The evolution of gene regulation by transcription factors and microRNAs. Nat Rev Genet 2007, 8(2):93-103.

53. Tsang J, Zhu J, van Oudenaarden A: MicroRNA-Mediated Feedback and Feedforward Loops Are Recurrent Network Motifs in Mammals. Molecular Cell 2007, 26(5):753-767.

54. Livak KJ, Schmittgen TD: Analysis of relative gene expression data using real-time quantitative PCR and the 2(-Delta Delta C(T)) Method. Methods 2001, 25(4):402-408.

55. Irizarry RA, Bolstad BM, Collin F, Cope LM, Hobbs B, Speed TP: Summaries of Affymetrix GeneChip probe level data. Nucleic Acids Res 2003, 31(4) e15.

56. Gentleman RC, Carey VJ, Bates DM, Bolstad B, Dettling M, Dudoit S, Ellis B, Gautier L, Ge Y, Gentry J, et al: Bioconductor: open software development for computational biology and bioinformatics. Genome Biol 2004, 5(10): R80.

57. Dai M, Wang P, Boyd AD, Kostov G, Athey B, Jones EG, Bunney WE, Myers RM, Speed TP, Akil H, et al: Evolving gene/transcript definitions significantly alter the interpretation of GeneChip data. Nucleic Acids Res 2005, 33(20):e175.

58. Smedley D, Haider S, Ballester B, Holland R, London D, Thorisson G, Kasprzyk A: BioMart-biological queries made easy. BMC Genomics 2009, $10: 22$.

59. Griffiths-Jones S, Saini HK, Dongen SV, Enright AJ: miRBase: tools for microRNA genomics. Nucleic Acids Res 2008, , 36 Database: D154-D158.

60. Chang WC: On Using Principal Components Before Separating a Mixture of Two Multivariate Normal Distributions. App/ Statist 1983, 32(3):267-275.

61. Yeung KY, Ruzzo WL: Principal component analysis for clustering gene expression data. Bioinformatics 2001, 17(9):763-774.

62. Hyvärinen A, Oja E: Independent component analysis: algorithms and applications. Neural Netw 2000, 13(4-5):411-430.

63. Hyvärinen A: Fast and Robust Fixed-Point algorithms for Independent Component Analysis. IEEE Trans on Neural Networks 1999, 10(3):626-634.

doi:10.1186/1471-2164-12-97

Cite this article as: Bang-Berthelsen et al.: Independent component and pathway-based analysis of miRNA-regulated gene expression in a model of type 1 diabetes. BMC Genomics 2011 12:97.

\section{Submit your next manuscript to BioMed Central and take full advantage of:}

- Convenient online submission

- Thorough peer review

- No space constraints or color figure charges

- Immediate publication on acceptance

- Inclusion in PubMed, CAS, Scopus and Google Scholar

- Research which is freely available for redistribution

Submit your manuscript at www.biomedcentral.com/submit
Biomed Central 